\title{
LncRNA SNHG14 regulates the DDP- resistance of non-small cell lung cancer cell through miR-133a/HOXB13 pathway
}

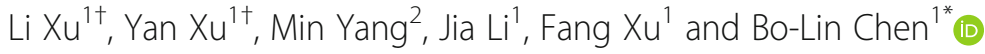

\begin{abstract}
Background: Recently, long non-coding RNAs (IncRNAs) have been reported to be involved in regulating chemoresistance of NSCLC, however, the role of InCRNA SNHG14 in the DDP-resistance of NSCLC remains unexplored.

Methods: Relative expression of SNHG14, HOXB13 and miR-133a in DDP-resistant A549 (A549/DDP) cell and its parental cell A549 were measured using qRT-PCR. Cell proliferation viability of indicated A549/DDP cell was estimated via CCK-8 and colony formation experiments. Cell cycle and apoptosis were analyzed through flow cytometry. Expression of apoptosis-related protein and HOXB13 were detected via western blot. The interaction among SNHG14, HOXB13 and miR-133a was predicted by bioinformatics and validated by dual-luciferase reporter assay.

Results: LnCRNA SNHG14 and HOXB13 were upregulated while miR-133a was downregulated in A549/DDP cell line compared to A549 cell line. SNHG14 knockdown or miR-133a overexpression was demonstrated to increase the DDPsensitivity of A549/DDP cells. SNHG14 was revealed to compete with HOXB13 for miR-133a binding in A549/DDP cells. Inhibition of miR-133a in A549 cells could reverse the promotive effects of SNHG14 knockdown on DDP-sensitivity, as well as the inhibitory effects on HOXB13 expression. HOXB13 overexpression was revealed to abolish the enhanced effects of miR-133a on the sensitivity of A549/DDP cell to DDP.

Conclusion: Our findings demonstrated that SNHG14 was involved in the development of DDP-resistance of A549/ DDP cells through miR-133a/HOXB13 axis, which may present a path to novel therapeutic stratagems for DDP resistance of NSCLC.
\end{abstract}

Keywords: NSCLC, LnCRNA SNHG14, MiR-133a, HOXB13; DDP-resistance

\section{Highlights}

1. LncRNA SNHG14 knockdown and miR-133a overexpression increased the DDP-sensitivity of A549/ DDP cell;

2. LncRNA SNHG14 targeted and inhibited miR-133a;

\footnotetext{
*Correspondence: chenbolin1009@163.com

${ }^{\dagger} \mathrm{Li} \mathrm{Xu}$ and Yan Xu are co-first authors.

${ }^{1}$ Thoracic Medicine Department 2, The Affiliated Cancer Hospital of Xiangya School of Medicine and Hunan Cancer Hospital, Central South University, No. 283, Tongzipo Road, Yuelu District, Changsha 410013, Hunan Province, PR China

Full list of author information is available at the end of the article
}

3. LncRNA SNHG14 regulates the DDP-resistance of non-small cell lung cancer cell through miR-133a/ HOXB13 pathway

\section{Background}

Lung cancer is one of the most common malignancies in the world that responsible for a considerable proportion cancer death [1]. The high mortality of lung cancer is largely due to the common diagnosis at advanced stages that impedes the curative treatment [2]. Without effective measures for early diagnosis where the tumors are still operable, and the lack of therapeutic options when

C The Author(s). 2020 Open Access This article is licensed under a Creative Commons Attribution 4.0 International License, which permits use, sharing, adaptation, distribution and reproduction in any medium or format, as long as you give appropriate credit to the original author(s) and the source, provide a link to the Creative Commons licence, and indicate if changes were made. The images or other third party material in this article are included in the article's Creative Commons licence, unless indicated otherwise in a credit line to the material. If material is not included in the article's Creative Commons licence and your intended use is not permitted by statutory regulation or exceeds the permitted use, you will need to obtain permission directly from the copyright holder. To view a copy of this licence, visit http://creativecommons.org/licenses/by/4.0/ The Creative Commons Public Domain Dedication waiver (http://creativecommons.org/publicdomain/zero/1.0/) applies to the data made available in this article, unless otherwise stated in a credit line to the data. 
the tumor is inoperable. These are the reasons why the five-year survival rate of NSCLC patients is less than $20 \%$ [3]. Cisplatin (DDP) is used as a first-line agent in the treatment of those postoperative or inoperable NSCLC patients [4]. The cancer-related death was previously demonstrated to decrease in the NSCLC patients who received DDP chemotherapy for 5 years compared to those untreated patients [5]. Nevertheless, individuals differently respond to DDP therapy and continuous DDP administration usually results in the occurrence of chemo-resistance, which frequently failed the clinical treatment [6]. Nowadays, DDP resistance is considered to be one of the most important impediments to the therapy of NSCLC patients.

MicroRNA (miRNA), featured by the short sequence (approximately 18-23 nucleotides), is a common subtype of endogenous non-coding RNA molecules [7]. The implication of miRNAs in modulating tumor-related gene expression have been well documented by numerous researches, showing it may function as a tumor repressor or an oncogene [8]. Accumulating data suggest that the tumor DDP-resistance could be modified by miRNAs [9], lighting up a novel research direction for tumor cell chemo-resistance. MiR-133a was revealed to be downregulated in NSCLC, and its expression level was negatively correlated with the lymphatic metastasis, tumor volume and clinical TNM stages [10]. In functional level, miR-133a was demonstrated to repress NSCLC cell proliferation and invasion in vitro $[11,12]$, however, up to now, there still has no study is performed to investigate whether miR-133a involves in the emergence of NSCLC chemoresistance. Our preliminary bioinformatics experimental results predicted that HOXB13 could be targeted by miR-133a. Furthermore, HOXB13 is a specific transcription factor of prostate-lineage that predominately existed in the tail bud in the development of embryonic stage [13]. Recently, HOXB13 was reported to confer the DDP-resistance to lung cancer cells through networking with ABCG1/EZH2/Slug [14]. However, the molecular mechanisms of HOXB13 in DDP-resistance of NSCLC cells involved are still unclear.

As another important subtype of non-coding RNAs, long non-coding RNA (lncRNA) with more than $200 \mathrm{nu}$ cleotides has also revealed to affect the chemo-resistance of NSCLC through multiple mechanisms $[15,16]$. Recently, lncRNA SNHG14 silencing was reported to repress NSCLC progression and enhance the NSCLC cell sensitivity to DDP [17]. According to bioinformatics prediction, lncRNA SNHG14 was a target of miR-133a.Therefore, we aimed to investigate whether lncRNA SNHG14 involves in the DDP-resistance of NSCLC cells by interacting with miR-133a/HOXB13 axis.

\section{Methods}

\section{NSCLC cell lines and transfection}

The parental NSCLC cell (A549) and DDP-resistant NSCLC cell (A549/DDP) were purchased from Cell bank of Chinese Academy of Sciences (Shanghai, China). Both A549 and A549/DDP cells were maintained in DMEM supplemented with fetal bovine serum (10\%, Sigma, USA), penicillin (100 U/ml, Sigma) and streptomycin $(100 \mathrm{mg} / \mathrm{ml}$, Sigma) under 5\% CO2 and 95\% air. Thee specific sh-SNHG14, miR-133a mimics, miR-133a inhibitor, pcDNA3.1-HOXB13 (pc-HOXB13), and corresponding negative control (NC) were obtained from GenePharma (Shanghai, China). All these oligonucleotides were transfected into A549/DDP cells using Lipofectamine 3000 (Invitrogen, USA) following the instructions of manufacturers. After $48 \mathrm{~h}$ of transfection, cells were harvested for further research.

\section{Quantitative real-time PCR (qRT-PCR) analysis}

TRIzol reagent (Invitrogen) was adopted to extract RNAs from indicated cells were prepared following the manufacturers' protocol. The RNA quality was determined via a NanoDrop 2000 instrument (Thermo Scientific, USA), and $5 \mu \mathrm{g}$ RNA was used as template to synthesis cDNA using a High-Capacity cDNA Reverse Transcription kit (Applied Biosystems, USA). SYBR Premix Ex Taq Kit (Takara, Tokyo) was adopted to conduct qRT-PCR on an ABI Prism 7700 system (PE Applied Biosystems, USA). Fold changes in RNA relative expression was counted through the $2^{-\Delta \Delta \mathrm{Ct}}$ method. Primers were summarized as follows:

\section{SNHG14: forward, 5 '-GGGTGTTTACGTAGACCA GAACC-3'; reverse, 5' -CTTCCAAAAGCCTTCTGC CTTAG-3' \\ miR-133a: forward, $5^{\prime}$-CTGCAGCTGGAGAGTGTG CG-3'; reverse, 5' -GTGCTCTGGAGGCTAGAGGT-3' HOXB13: forward, 5' -ATGGAGCCCGGCAATTAT GCCACC-3'; reverse, 5' -TTAAGGGGTAGCGCTG TTCTT-3' \\ GAPDH: forward, 5'-GGCGTTCTCTTTGGAAAG GTGTTC-3'; reverse, 5' - GTACTCAGCGGCCAGC ATCG -3 \\ U6: forward 5'-CTC GCT TCG GCA GCA CA-3', re- verse $5^{\prime}$-AAC GCT TCA CGA ATT TGC GT-3'}

\section{Cell proliferation viability analysis (CCK-8 and colony formation)}

After $48 \mathrm{~h}$ of transfection, exponentially growing A549/ DDP cells $\left(1 \times 10^{5}\right.$ cells/well $)$ were harvested and plated into 96-well plates supplemented with $\operatorname{DDP}(0,2,4,6$, AND $8 \mu \mathrm{g} / \mathrm{ml}$ ), and then the proliferation viability of A549/DDP cells were tested by the Cell Counting Kit-8 (CCK-8; Dojindo Molecular Technologies, Japan) and colony formation assays after $24 \mathrm{~h}$ of DDP incubation. For CCK-8, the CCK-8 solution $(200 \mu \mathrm{l})$ was added to the each well and then incubated at $37^{\circ} \mathrm{C}$ for $2 \mathrm{~h}$. The absorbance was measured by a microplate reader (Bio- 
Rad Laboratories, USA) at $450 \mathrm{~nm}$. For colony formation assay, indicated A549/DDP cells (2000 cells/dish) were seeded into $35-\mathrm{mm}$ culture dish and maintained at $37^{\circ} \mathrm{C}$. After 2 weeks, the visible colonies were fixed in $4 \%$ paraformaldehyde for $0.5 \mathrm{~h}$, stained with $0.1 \%$ crystal violet solution for $10 \mathrm{~min}$, and counted using a microscope.

\section{Cell cycle and apoptosis analysis}

The A549/DDP cell cycle and apoptosis were estimated using flow cytometry. In brief, $48 \mathrm{~h}$ after transfection, cells were harvested for DDP treatment for additional $24 \mathrm{~h}$. For the cell cycle assay, A549/DDP cells were fixed in $75 \%$ alcohol for $30 \mathrm{~min}$, and then stained with propidium idoide (Sigma, MO, USA) for $15 \mathrm{~min}$ in dark. For cell apoptosis analysis, A549/DDP cells were washed with PBS followed by staining with Annexin V/PI kit (Vazyme, Nanjing, China) following the instructions of manufacturer. Finally, a BD Biosciences FACSCalibur Flow Cytometer (BD Biasciences, USA) was applied to detect the cell cycle and apoptosis.

\section{Dual-luciferase reporter assay}

The wide type and mutant type miR-133a binding sequence of IncRNA SNHG14 and HOXB13 mRNA 3'UTR were sub-cloned into the pmirGLO vector (Promega, Madison, USA) and named as SNHG14-WT, HOXB13-WT, SNHG14-MUT, and HOXB13-MUT, respectively. A549/DDP cells $\left(1 \times 10^{6}\right.$ cells/wel $)$ seeded in 96-well plates were co-transfected with constructed recombinant luciferase vectors and miR-133a mimics or mimics NC using Lipofectamine 3000 (Invitrogen). Luciferase intensity of A549/DDP cell was examined using the Dual-Luciferase Reporter Assay System (Promega) after $48 \mathrm{~h}$ of co-transfection.

\section{Western blot assay}

Total proteins of indicated A549/DDP cells were isolated using the RIPA buffer (Beyotime, Beijing, China) contained protease inhibitors (Roche, Germany). Protein samples $(50 \mu \mathrm{g})$ were isolated via 10\% SDS-PAGE and then transferred into polyvinylidene difluoride (PVDF) membranes (Millipore, UK). After incubated in 5\% skimmed milk for $2 \mathrm{~h}$, the membranes were subjected for probe of primary antibodies that against Bcl-2 (1: 2000, ab491583, Abcam), Bax (1:1000, ab199677, Abcam), cleaved-caspase-3 (1:1000, orb227889, Biorbyt) and HOXB13 (1:2000, ab53931, Abcam) overnight. Subsequently, the membranes were washed with PBS and then probed with corresponding secondary antibodies conjugated with horseradish peroxidase for $2 \mathrm{~h}$. The signals were visible using an enhanced chemiluminescent reagent (ECL, Germany).

\section{Statistical analysis}

All experiments were performed at least three times. Data were presented as mean \pm standard deviation (SD), and all statistical analyses were conducted on SPSS (19.0 vision, IBM). Student's t test or one-way analysis of variance followed by Tukey post hoc test was applied for the analysis of the difference between two or more groups. $P$ value less than 0.05 was considered significant.

\section{Results}

LncRNA SNHG14 and HOXB13 were highly expressed, while miR-133a was lowly expressed in DDP-resistant NSCLC cell

To investigate whether IncRNA SNHG14, HOXB13 and miR-133a play roles in the chemo-resistance of NSCLC, we firstly examined their expression in parental NSCLC cell (A549) and DDP-resistant NSCLC cell (A549/DDP) using qRT-PCR. As results indicated that lncRNA SNHG14 and HOXB13 were remarkably increased, while miR-133a was significantly decreased in A549/ DDP cells compared with A549 cells (Fig. 1a). The dysregulation of lncRNA SNHG14, HOXB13 and miR-133a in A549/DDP cells implied that they may be involved in the chemo-resistance of NSCLC.

\section{LncRNA SNHG14 silencing enhanced the sensitivity of A549/DDP cell to DDP}

To further investigate the functional roles of IncRNA SNHG14 in the development of DDP resistance of A549/DDP cells, we silenced its expression in A549/ DDP cells followed by the examination of cell viability, proliferation cycle and apoptosis. Results from qRT-PCR A549/DDP indicated a high knockdown efficiency of shSNHG14 in A549/DDP cells (Fig. 2a). The effects of A549/DDP cell viability under the treatment of DDP with different concentration $(0,2,4,6$ and $8 \mu \mathrm{g} / \mathrm{ml})$ were evaluated by CCK-8. The DDP-induced downregulation

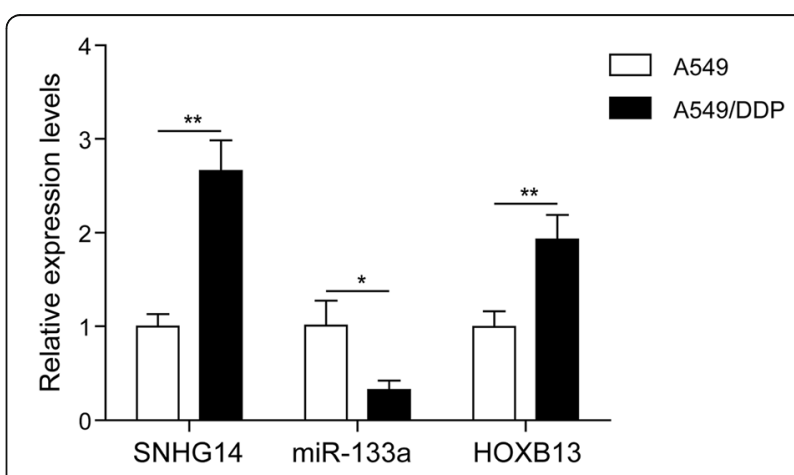

Fig. 1 LnCRNA SNHG14 and HOXB13 were highly expressed, while miR-133a was lowly expressed in DDP-resistant NSCLC cell. a Levels of IncRNA SNHG14, HOXB and miR-133a in normal A549 and DDPresistant A549 (A549/DDP) cells were examined using qRT-PCR. ${ }^{*} P<0.05,{ }^{*} P<0.01$ 
of A549/DDP cell viability was augmented in shSNHG14 group (Fig. 2b). Moreover, results from colony formation experiment demonstrated that IncRNA SNHG14 knockdown significantly reduced the A549/DDP cell number under the treatment of DDP $(1 \mu \mathrm{g} / \mathrm{ml})$ (Fig. 2c). In addition, flow cytometry was conducted in lncRNA SNHG14 silenced A549/DDP cells to estimate the influences of SNHG14 knockdown on A549/DDP cell cycle and apoptosis. In the presence of $1 \mu \mathrm{g} / \mathrm{ml}$ of DDP, lncRNA SNHG14 silencing resulted in a significant upregulation of cell number in G0/G1 phases and a remarkable downregulation of S phase (Fig. 2d). The A549/DDP cell apoptosis rate was significantly increased in sh-SNHG14 transfected group (Fig. 2e). In the western blot detection of apoptosisrelated protein expression, we found that sh-SNHG14 transfection significantly decreased $\mathrm{Bcl}-2$ expression, while increased Bax and cleaved-caspase-3 expression (Fig. 2f). Taken together, these findings strongly supported that lncRNA SNHG14 knockdown enhanced the sensitivity of A549/DDP cells to DDP.

\section{miR-133a treatment enhanced the sensitivity of A549/ DDP cell to DDP}

We then explored the functional roles of miR-133a in the development of DDP resistance of NSCLC by overexpressing its expression in A549/DDP cells, and then estimated the cell viability, proliferation, cycle and apoptosis of miR-133a overexpressed A549/DDP cells. The overexpression efficiency of miR-133a in A549/DDP cells was determined by qRT-PCR (Fig. 3a). CCK-8 was utilized to detect the influences of miR-133a overexpression on A549/DDP cell viability in the presence of different concentrations of DDP $(0,2,4,6$ and $8 \mu \mathrm{g} / \mathrm{ml})$. As results showed that the A549/DDP cell viability of miR133a mimics group was significantly lower than that of sh-NC group (Fig. 3b). Forthermore, miR-133a overexpression led to a significant reduction of A549/DDP cell number (Fig. 3c). In addition, the influences of miR133a mimics treatment on A549/DDP cell cycle and apoptosis in the presence of $1 \mu \mathrm{g} / \mathrm{ml}$ of DDP were estimated via flow cytometry. As results demonstrated that miR-133a overexpression remarkably increased the A549/DDP cell number of G0/G1 phases, while reduced the A549/DDP cell number of S phase (Fig. 3d). Moreover, miR-133a overexpression led to a significant upregulation of A549/DDP cell apoptosis rate induced by DDP (Fig. 3e). Similar to lncRNA SNHG14 knockdown, we also found that miR-133a overexpression significantly downregulated the protein expression of Bcl-2 while upregulated the protein expression of $\mathrm{Bax}$ and cleavedcaspase-3 in A549/DDP cells treated with DDP (Fig. 3f). These data highly suggested that miR-133a overexpression increased the DDP sensitivity of A549/DDP cells.

\section{LncRNA SNHG14 competed with HOXB13 for miR-133a binding}

Numerous studies have shown that lncRNAs participated almost in every aspect of the biological processes of tumor progression by competitively binding to specific miRNAs and thus releasing target genes. Therefore, bioinformatics analysis was performed to predict the target miRNAs which could interact with lncRNA SNHG14. We found that lncRNA SNHG14 possessed complementary sequence of miR-133a. Moreover, HOXB13 was also found to possess the complementary sequence of miR-133a (Fig. 4a). To validate the direct binding between IncRNA SNHG14 and miR-133a, as well as miR-133a and HOXB13, dualluciferase reporter assay was conducted in A549/DDP cells. Results demonstrated that co-transfection with SNHG14WT or HOXB13-WT and miR-133a mimics caused a significant repression of luciferase activity, while co-transfection with SNHG14-MUT or HOXB13-MUT and miR-133a mimics exhibited no obvious effects on the luciferase activity (Fig. 4b). Besides, we found thatA549/DDP lncRNA SNHG14 knockdown resulted in an upregulation of miR133a in A549/DDP cells (Fig. 4c), and miR-133a overexpression caused a downregulation of HOXB13 (Fig. 4d). Together, these results indicated that IncRNA SNHG14 competed with HOXB13 for miR-133a binding.

HOXB13 overexpression reversed the enhanced effects of miR-133a on the sensitivity of A549/DDP cell to DDP

To investigate whether HOXB13 plays a role in the enhanced effects of miR-133a on the sensitivity of A549/ DDP cell to DDP, we treated A549/DDP cells with pcHOXB13 and miR-133a mimics followed by the examination of cell viability, proliferation and apoptosis in the presence of DDP. The results indicated that overexpression of HOXB13 dramatically increased A549/DDP cell viability and colony-formation ability. However, cotransfection of pc-HOXB13 and miR-133a mimics reversed the upregulation of cell viability and colonyformation ability induced by pc-HOXB13 transfection in A549/DDP cells (Fig. 5a-b). Moreover, by using flow cytometry analysis, we revealed that pc-HOXB13 transfection sharply reduced A549/DDP cell apoptosis, while cotransfection of pc-HOXB13 and miR-133a mimics abolished the reduction of A549/DDP cell apoptosis induced by pc-HOXB13 transfection (Fig. 5c). Additionally, results from western blot indicated that overexpression of HOXB13 dramatically increased the expression of HOXB13 and Bcl-2 while decreased the expression of Bax and cleaved caspase- 3 in the A549/DDP cells, however, co-transfection of pc-HOXB13 and miR-133a mimics abolished the dysregulation of these molecules (Fig. 5d). These findings suggested that overexpression of HOXB13 repressed the sensitivity of A549/DDP cell to DDP through antagonizing miR-133a. 


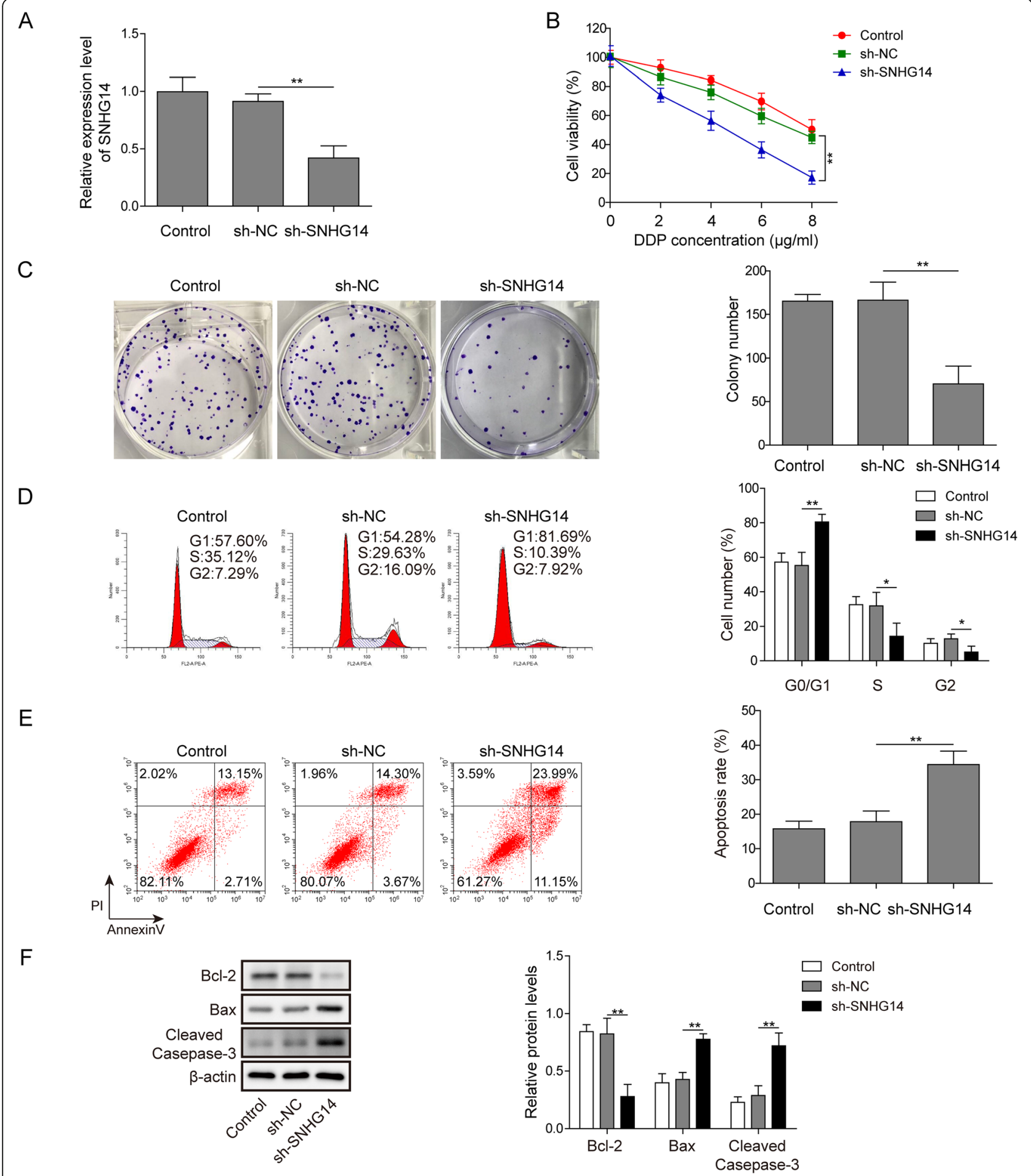

Fig. 2 LncRNA SNHG14 silencing enhanced the sensitivity of A549/DDP cell to DDP. a A549/DDP cells transfected with sh-NC or sh-SNHG14. qRTPCR examined expression of InCRNA SNHG14A549/DDP. $\mathbf{b}$ After transfected with sh-NC and sh-SNHG14, the cell viability of A549/DDP cell was detected via CCK-8 under the treatment of DDP with different concentration $(0,2,4,6$ and $8 \mu \mathrm{g} / \mathrm{ml})$. c The cell proliferation of sh-NC and shSNHG14 treated A549/DDP cell was detected by colony formation experiment in the presence of DDP (1 $\mu \mathrm{g} / \mathrm{ml})$. $\mathbf{d}$ and e After $48 \mathrm{~h}$ of shSNHG14 transfection, the A549/DDP cell was treated with DDP $(1 \mathrm{\mu g} / \mathrm{ml})$ and then subjected for cell cycle and apoptosis detection using flow cytometry analysis. $\mathbf{f}$ Protein expression levels of Bcl-2, Bax and cleaved-caspase-3 were measured by western blot in IncRNA SNHG14 blocked A549/DDP cells treated with DDP $(1 \mu \mathrm{g} / \mathrm{ml})$ 


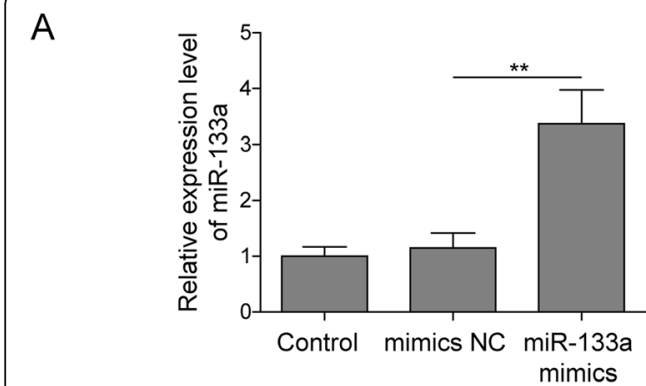

C

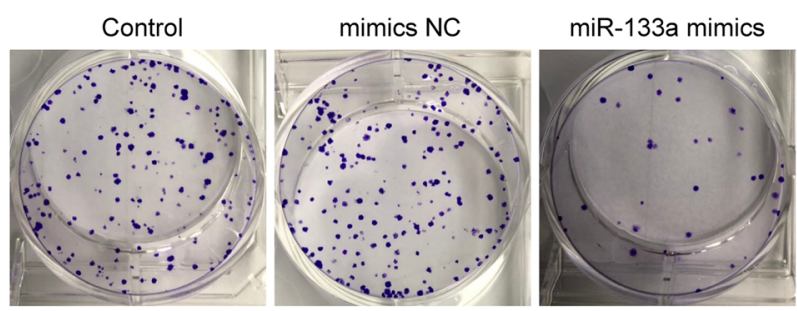

D

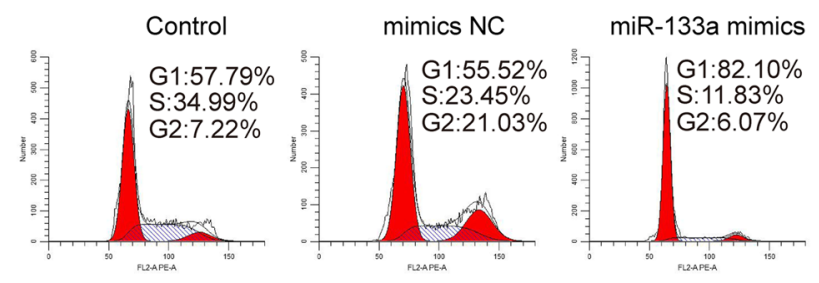

E
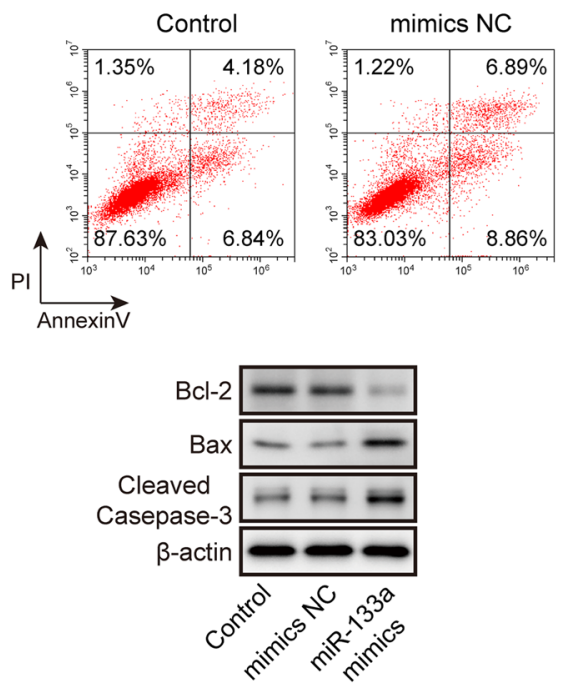

F

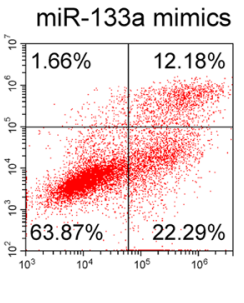

B
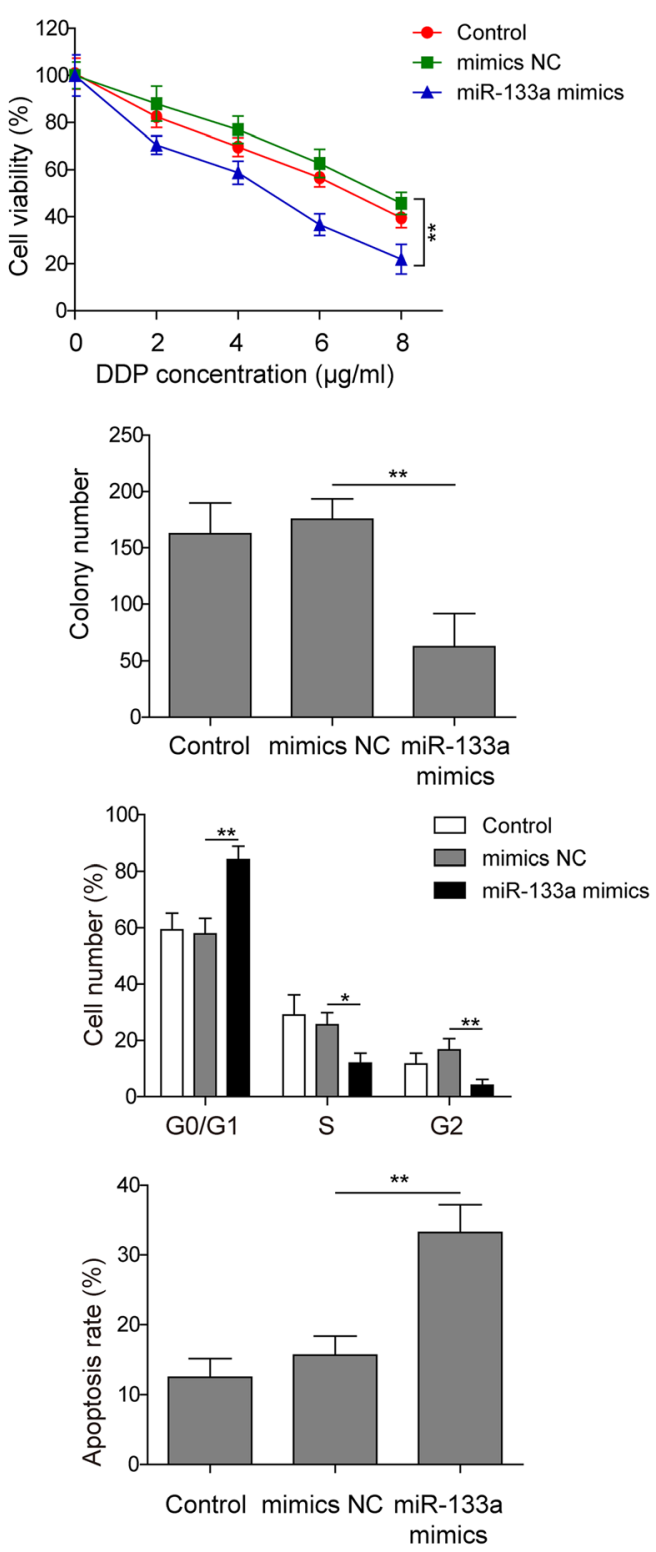

mimics

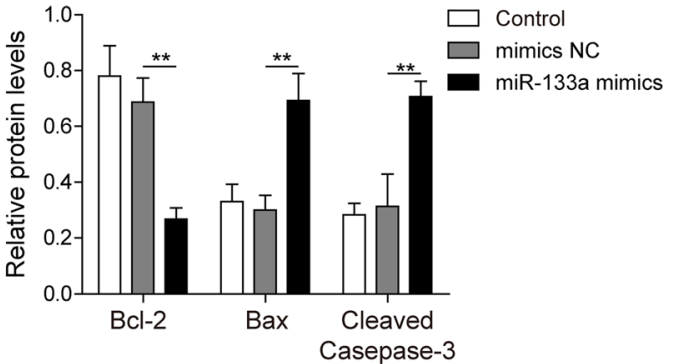

Fig. 3 miR-133a treatment enhanced the sensitivity of A549/DDP cell to DDP. a qRT-PCR was conducted in mimics NC and miR-133a mimics transfected A549/DDP cells to examine the overexpression efficiency of miR-133a. b After transfected with mimics NC and miR-133a mimics for $48 \mathrm{~h}$, the cell viability of A549/DDP cell was examined in the presence of DDP $(1 \mu \mathrm{g} / \mathrm{ml})$ by CCK-8. c The cell proliferation of mimics NC and miR-133a mimics treated A549/DDP cell was detected by colony formation experiment in the presence of DDP $(1 \mathrm{\mu g} / \mathrm{ml})$. $\mathbf{d}$ and $\mathbf{e}$ After $48 \mathrm{~h}$ of mimics NC and miR-133a mimics transfection, the A549/DDP cell was treated with DDP $(1 \mu \mathrm{g} / \mathrm{ml})$ and then subjected for cell cycle and apoptosis detection using flow cytometry analysis. $\mathbf{f}$ Protein expression levels of Bcl-2, Bax and cleaved-caspase-3 were measured by western blot in miR-133a mimics transfected A549/DDP cells treated with DDP (1 $\mathrm{\mu g} / \mathrm{ml})$ 


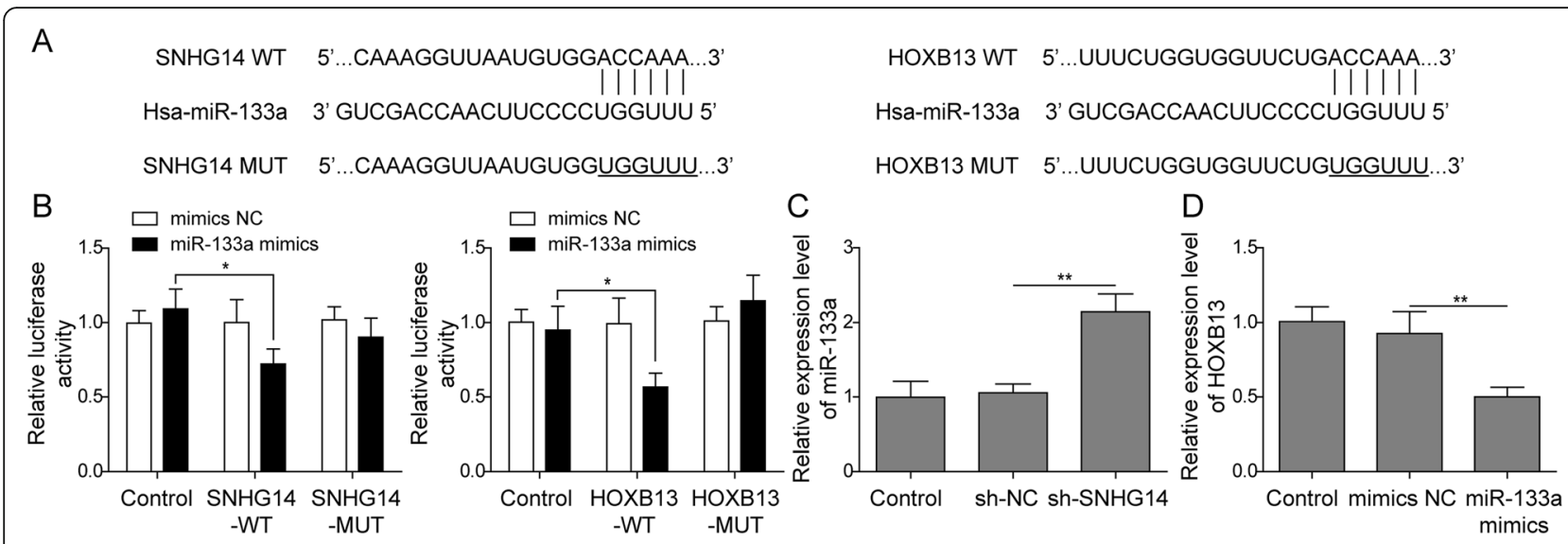

Fig. 4 LnCRNA SNHG14 competed with HOXB13 for miR-133a binding. a Putative miR-133a binding sequence of IncRNA SNHG14 and HOXB13. b Dual-luciferase reporter experiment was conducted in A549/DDP cell to validate the interplay between SNHG14 and miR-133a, as well as miR133a and HOXB13. c miR-133a level was examined in sh-NC and sh-SNHG14 transfected A549/DDP cell by qRT-PCR. $\mathbf{d}$ qRT-PCR analysis of HOXB13 in mimics NC and miR-133a mimics transfected A549/DDP cell

miR-133a/HOXB13 pathway involved in the IncRNA SNHG14 regulation of A549/DDP cell sensitivity to DDP Considering the interaction among lncRNA SNHG14, HOXB13 and miR-133a in A549/DDP cells, we further investigated whether miR-133a is involved in the process of lncRNA SNHG14 regulating the DDP-resistance of NSCLC through HOXB13 pathway. We have shown that sh-SNHG14 transfection could significantly decrease the A549/DDP cell viability in the presence of DDP while this repression of cell viability caused by sh-SNHG14 treatment was reversed by the miR-133a inhibitor (Fig. 6a). Similarly, in colony formation assay, the sh-SNHG14 treatment induced reduction of A549/DDP cell number was recovered by the cotransfection of sh-SNHG14 and miR-133a inhibitor (Fig. 6b). Moreover, the sh-SNHG14 treatment induced upregulation of G0/G1 phases cell number and downregulation of S phase cell number were counteracted partially by inhibition of miR-133a (Fig. 6c). The co-transfection of sh-SNHG14 and miR-133a inhibitor also abolished the promotive effects of sh-SNHG14 transfection on A549/DDP cell DDP-inducted apoptosis (Fig. $6 \mathrm{~d})$. In the western blot assay, we demonstrated that cotransfection of sh-SNHG14 and miR-133a inhibitor could reverse the sh-SNHG14 transfection induced Bcl-2 downregulation and Bax, cleaved-caspas-3 upregulation (Fig. 6e). In addition, we found that lncRNA SNHG14 knockdown significantly reduced the epression of HOXB13, however, cotransfection of sh-SNHG14 and miR-133a inhibitor abolished this effect (Fig. 6e). These results implied that miR-133a/ HOXB13 pathway involved in the IncRNA SNHG14 regulation of A549/DDP cell sensitivity to DDP.

\section{Discussion}

Although the exact mechanisms underlying DDPresistance remain largely unclear, it is certain that this problem could not be conquered through targeting any single mechanism strategy $[18,19]$. Galuzzi et al. have proposed four distinct DDP-resistance mechanisms: (i), by reducing the cellular concentration of DDP to prevent its binding with DNA; (ii), repairing the DDP-DNA adducts; (iii), restoring the dysregulated signaling pathways in response to DNA damage caused by DDP; (iiii), through indirect mechanisms that do not involve DDPrelated signals but confer resistance to DDP-induced death [20]. Considering the substantial genes, proteins and signal cascades involved in the emergence of DDPresistance [21], it is likely to fail if we focus on any single mechanism-targeted strategy. Here, we demonstrated that IncRNA SNHG14, through miR-133a/HOXB13 pathway, played as a regulator during the development of DDP-resistance of NSCLC cells, providing a novel signal cascade that may be used as a promising therapeutic target for the overcome of DDP-resistance of NSCLC.

With the advancement of technologies in DNA sequencing and bioinformatics analysis, numerous of lncRNAs and miRNAs have been proven to be involved in the drug-resistance of NSCLC through multiple mechanisms [16, 22]. For example, Ma LY et al. have shown the repressive effects of IncRNA TRPM2-AS knockdown on NSCLC cell DDP-resistance, moreover, they also demonstrated this effects was p53- p66shc pathway dependent [23]. Moreover, miR-488 was revealed to repress NSCLC cell proliferation and reduce NSCLC cell sensitivity to DDP via the activation of eIF3a-mediated NER signaling cascade [24]. LncRNA SNHG14 participates in the progression of various human tumors, such as glioma, cervical cancer and NSCLC [25-27]. Recently, it was also involved in the drugresistance of tumor cells. Dong $\mathrm{H}$ et al. reported that SNHG14 enhanced the trastuzumab resistance of breast cancer cells by modulating PABPC1 level trough H3K27 


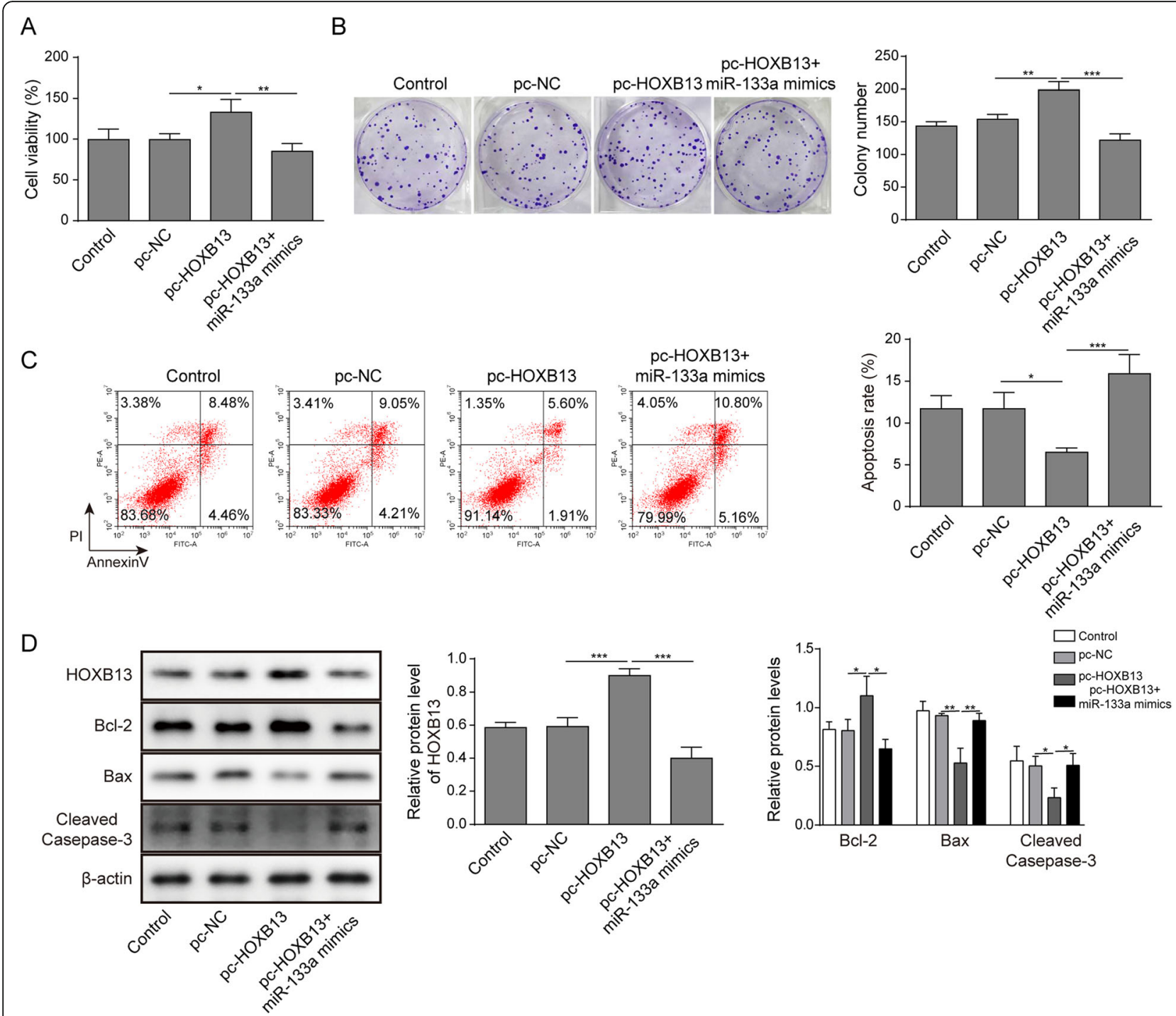

Fig. 5 HOXB13 overexpression reversed the enhanced effects of miR-133a on the sensitivity of A549/DDP cell to DDP. A549/DDP cells under DDP treatment $(1 \mu \mathrm{g} / \mathrm{ml})$ were co-transfected with pc-HOXB13 and miR-133a mimics, then the cell viability, proliferation, and apoptosis was determined by $\mathbf{a}$ CCK-8, $\mathbf{b}$ colony formation and $\mathbf{c}$ flow cytometry analysis, respectively. $\mathbf{d}$ The protein expression of HOXB13, Bax, BCl-2 and caspase-3 in pc-HOXB13 and miR-133a mimics co-transfected A549/DDP cells were assessed by western blot

acetylation [28]. Moreover, SNHG14 was proved to confer gefitinib resistance in NSCLC cell by increasing ABCB1 level through sponging miR-206-3p [29]. In line with previous researches, our study found a significant upregulation of lncRNA SNHG14 in A549/DDP cell, and SNHG14 knockdown resulted in an enhancement of DDP-sensitivity of A549/DDP cell.

It was reported that miR-133a could increase the DDP-sensitivity of Hep-2 and vincristine resistant Hep-2v cell through reducing ATP7B level [30]. Although miR-133a plays a role in the multiple biological processes of NSCLC cell, including proliferation, migration and invasion [11, 12], its implication in NSCLC DDP-resistance remains no report. In this study, we found miR-133a was downregulated in DDP-resistant A549/DDP cells, and its overexpression increased the DDP-sensitivity of A549/DDP cells. Taken together, lncRNA SNHG14 and miR-133a were involved in the DDP-resistance of NSCLC.

The involvement of lncRNAs in tumor cell drugresistance is largely mediated by miRNAs and thus affected their downstream target genes [31]. For instance, LncRNA SNHG14 was demonstrated to increase gemcitabine resistance of pancreatic cancer cells by interacting with miR-101 [32]. In the present study, IncRNA SNHG14 was firstly found to compete with HOXB13 for miR-133a binding. Functionally, 


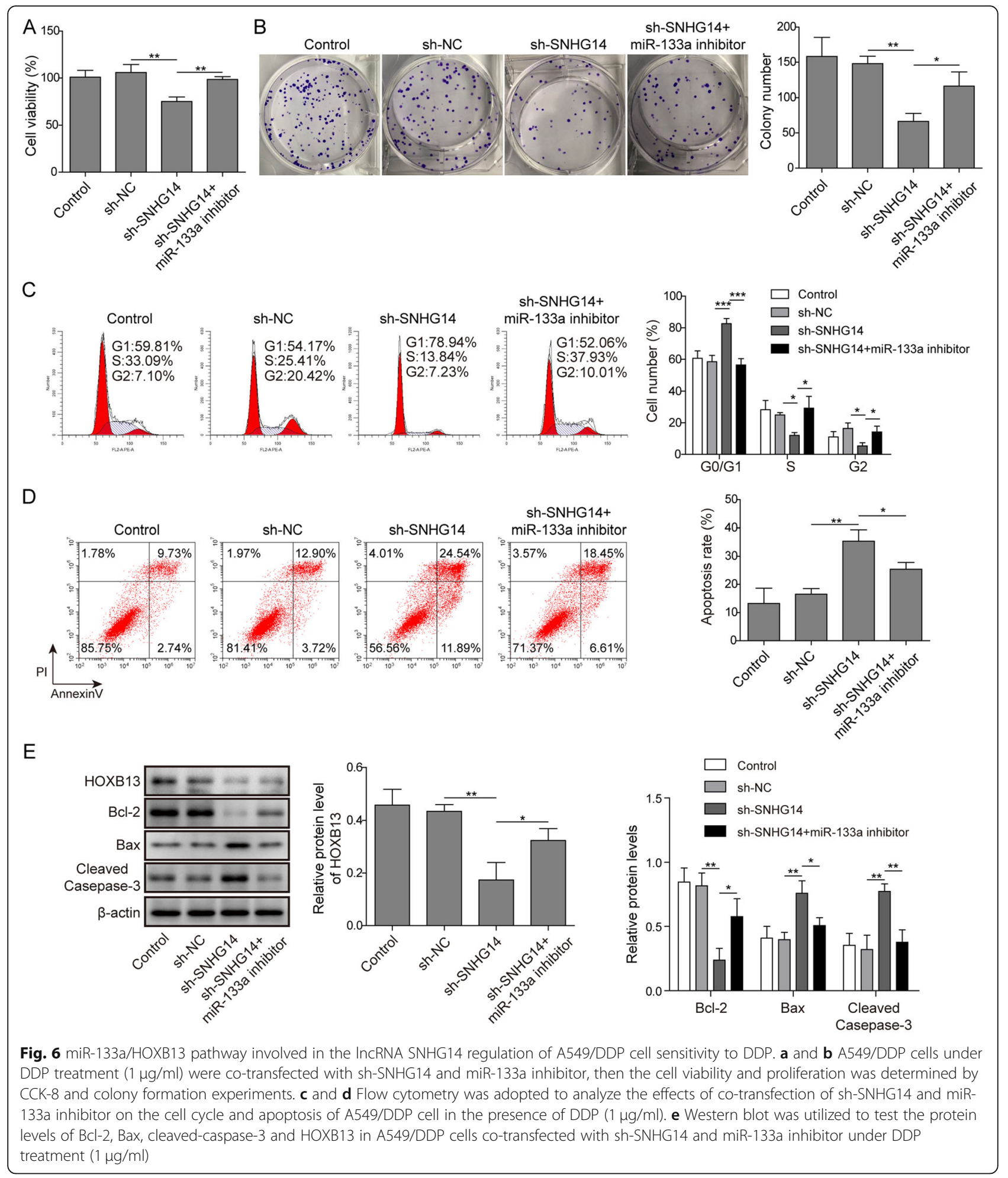

miR-133a inhibition abolished the repressive effects of sh-SNHG14 on DDP-resistance and HOXB13 expression. Moreover, HOXB13 overexpression reversed the enhanced effects of miR-133a on the sensitivity of A549/DDP cell to DDP.

\section{Conclusion}

In conclusion, our findings provided evidences that lncRNA SNHG14 regulated the DDP-resistance of NSCLC cell in vitro by increasing HOXB13 expression through miR-133a. Thus, IncRNA SNHG14/ 
miR-133a/HOXB13 regulatory network might be promising therapeutic target for NSCLC drugresistance.

\section{Abbreviations}

LncRNAs: Long non-coding RNAs; NSCLC: Non-small cell lung cancer; SCLC: Small-cell lung cancer; MicroRNAs: MiRNAs; Lnc RNA SNHG14: LncRNAsmall nucleolar RNA host gene 14; HOXB13: Homeobox B13

\section{Acknowledgements}

Not Applicable.

\section{Authors' contributions}

guarantor of integrity of the entire study, study concepts, manuscript editing, manuscript review: BLC; study design: BLC, LX; definition of intellectual content: YX, MY; literature research, data acquisition, manuscript preparation: FX; experimental studies: BLC, LX, YX; data analysis: MY, JL; statistical analysis: YX, MY, JL. All authors have read and approved the manuscript.

\section{Funding}

None.

\section{Availability of data and materials}

All data generated or analyzed during this study are included in this published article.

\section{Ethics approval and consent to participate} Not applicable.

\section{Consent for publication}

The informed consent obtained from study participants.

\section{Competing interests}

The authors declare that they have no conflict of interest.

\section{Author details}

${ }^{1}$ Thoracic Medicine Department 2, The Affiliated Cancer Hospital of Xiangya School of Medicine and Hunan Cancer Hospital, Central South University, No. 283, Tongzipo Road, Yuelu District, Changsha 410013, Hunan Province, PR China. ${ }^{2}$ Respiratory Medicine Department 2, Hunan Children's Hospital, Changsha 410007, Hunan Province, PR China.

Received: 26 March 2020 Accepted: 31 August 2020 Published online: 15 October 2020

\section{References}

1. Woodard GA, Jones KD, Jablons DM. Lung cancer staging and prognosis. Cancer Treat Res. 2016;170:47-75.

2. Duruisseaux M, Esteller M. Lung cancer epigenetics: from knowledge to applications. Semin Cancer Biol. 2018;51:116-28.

3. O'Kane GM, Bradbury PA, Feld R, Leighl NB, Liu G, Pisters KM, Kamel-Reid S, Tsao MS, Shepherd FA. Uncommon EGFR mutations in advanced non-small cell lung cancer. Lung Cancer. 2017;109:137-44.

4. Dasari S, Tchounwou PB. Cisplatin in cancer therapy: molecular mechanisms of action. Eur J Pharmacol. 2014;740:364-78.

5. Pignon JP, Tribodet H, Scagliotti GV, Douillard JY, Shepherd FA, Stephens RI, Dunant A, Torri V, Rosell R, Seymour L, et al. Lung adjuvant cisplatin evaluation: a pooled analysis by the LACE collaborative group. J Clin Oncol. 2008:26(21):3552-9.

6. Fennell DA, Summers Y, Cadranel J, Benepal T, Christoph DC, Lal R, Das M, Maxwell F, Visseren-Grul C, Ferry D. Cisplatin in the modern era: the backbone of first-line chemotherapy for non-small cell lung cancer. Cancer Treat Rev. 2016;44:42-50.

7. Wojciechowska A, Braniewska A, Kozar-Kaminska K. MicroRNA in cardiovascular biology and disease. Adv Clin Exp Med. 2017;26(5):865-74.

8. Hassan N, Zhao JT, Sidhu SB. The role of microRNAs in the pathophysiology of adrenal tumors. Mol Cell Endocrinol. 2017;456:36-43.

9. Lin G, Xu K. Advances in tumor chemo-resistance regulated by MicroRNA. Zhongguo Fei Ai Za Zhi. 2014;17(10):741-9.

10. Lan D, Zhang X, He R, Tang R, Li P, He Q, Chen G. MiR-133a is downregulated in non-small cell lung cancer: a study of clinical significance. Eur J Med Res. 2015;20:50.
11. Xu M, Wang YZ. miR133a suppresses cell proliferation, migration and invasion in human lung cancer by targeting MMP14. Oncol Rep. 2013;30(3):1398-404.

12. Wang LK, Hsiao TH, Hong TM, Chen HY, Kao SH, Wang WL, Yu SL, Lin CW, Yang PC. MicroRNA-133a suppresses multiple oncogenic membrane receptors and cell invasion in non-small cell lung carcinoma. PLoS One. 2014;9(5):e96765.

13. Kim YR, Kim IJ, Kang TW, Choi C, Kim KK, Kim MS, Nam KI, Jung C. HOXB13 downregulates intracellular zinc and increases NF-kappaB signaling to promote prostate cancer metastasis. Oncogene. 2014;33(37):4558-67.

14. Zhan J, Wang P, Li S, Song J, He H, Wang Y, Liu Z, Wang F, Bai H, Fang W, et al. HOXB13 networking with ABCG1/EZH2/slug mediates metastasis and confers resistance to cisplatin in lung adenocarcinoma patients. Theranostics. 2019;9(7): 2084-99.

15. Sun W, Zu Y, Fu X, Deng Y. Knockdown of IncRNA-XIST enhances the chemosensitivity of NSCLC cells via suppression of autophagy. Oncol Rep. 2017; 38(6):3347-54.

16. Wang L, Ma L, Xu F, Zhai W, Dong S, Yin L, Liu J, Yu Z. Role of long noncoding RNA in drug resistance in non-small cell lung cancer. Thorac Cancer. 2018;9(7):761-8.

17. Jiao P, Hou J, Yao M, Wu J, Ren G. SNHG14 silencing suppresses the progression and promotes cisplatin sensitivity in non-small cell lung cancer. Biomed Pharmacother. 2019;117:109164.

18. Wang M, Liu ZM, Li XC, Yao YT, Yin ZX. Activation of ERK1/2 and Akt is associated with cisplatin resistance in human lung cancer cells. J Chemother. 2013;25(3):162-9.

19. Shtivelman E, Hensing T, Simon GR, Dennis PA, Otterson GA, Bueno R, Salgia R. Molecular pathways and therapeutic targets in lung cancer. Oncotarget. 2014;5(6):1392-433.

20. Galluzzi L, Vitale I, Michels J, Brenner C, Szabadkai G, Harel-Bellan A, Castedo M, Kroemer G. Systems biology of cisplatin resistance: past, present and future. Cell Death Dis. 2014:5:e1257.

21. Rotow J, Bivona TG. Understanding and targeting resistance mechanisms in NSCLC. Nat Rev Cancer. 2017;17(11):637-58.

22. Zang $\mathrm{H}$, Wang W, Fan $\mathrm{S}$. The role of microRNAs in resistance to targeted treatments of non-small cell lung cancer. Cancer Chemother Pharmacol. 2017;79(2):227-31

23. Ma LY, Xie XW, Ma L, Pang JL, Xiong XM, Zheng HD, Shen XL, Wen ZG, Wang HY. Downregulated long non-coding RNA TRPM2-AS inhibits cisplatin resistance of non-small cell lung cancer cells via activation of p53- p66shc pathway. Eur Rev Med Pharmacol Sci. 2017;21 (11):2626-34.

24. Fang $C$, Chen YX, Wu NY, Yin JY, Li XP, Huang HS, Zhang W, Zhou HH, Liu ZQ. MiR-488 inhibits proliferation and cisplatin sensibility in non-small-cell lung cancer (NSCLC) cells by activating the elF3a-mediated NER signaling pathway. Sci Rep. 2017;7:40384.

25. Wang Q, Teng Y, Wang R, Deng D, You Y, Peng Y, Shao N, Zhi F. The long noncoding RNA SNHG14 inhibits cell proliferation and invasion and promotes apoptosis by sponging miR-92a-3p in glioma. Oncotarget. 2018;9(15):12112-24.

26. Ji N, Wang Y, Bao G, Yan J, Ji S. LncRNA SNHG14 promotes the progression of cenvical cancer by regulating miR-206/WWHAZ. Pathol Res Pract. 2019;215(4):668-75.

27. Zhang Z, Wang Y, Zhang W, Li J, Liu W, Lu W. Long non-coding RNA SNHG14 exerts oncogenic functions in non-small cell lung cancer through acting as an miR-340 sponge. Biosci Rep. 2019;39(1):BSR20180941.

28. Dong $H$, Wang W, Mo S, Liu Q, Chen $X$, Chen R, Zhang Y, Zou K, Ye M, He $X$, et al. Long non-coding RNA SNHG14 induces trastuzumab resistance of breast cancer via regulating PABPC1 expression through H3K27 acetylation. J Cell Mol Med. 2018;22(10):4935-47.

29. Wu K, Li J, Qi Y, Zhang C, Zhu D, Liu D, Zhao S. SNHG14 confers gefitinib resistance in non-small cell lung cancer by up-regulating $A B C B 1$ via sponging miR-206-3p. Biomed Pharmacother. 2019;116:108995.

30. Wang $X$, Zhu W, Zhao X, Wang P. miR-133a enhances the sensitivity of Hep2 cells and vincristine-resistant Hep-2v cells to cisplatin by downregulating ATP7B expression. Int J Mol Med. 2016;37(6):1636-42.

31. Fang Z, Zhao J, Xie W, Sun Q, Wang H, Qiao B. LncRNA UCA1 promotes proliferation and cisplatin resistance of oral squamous cell carcinoma by sunppressing miR-184 expression. Cancer Med. 2017;6(12):2897-908.

32. Zhang $X$, Zhao $P$, Wang $C$, Xin B. SNHG14 enhances gemcitabine resistance by sponging miR-101 to stimulate cell autophagy in pancreatic cancer. Biochem Biophys Res Commun. 2019;510(4):508-14.

\section{Publisher's Note}

Springer Nature remains neutral with regard to jurisdictional claims in published maps and institutional affiliations. 